\title{
Holographic Rényi relative divergence in JT gravity
}

\section{Tomonori Ugajin}

Center for Gravitational Physics, Yukawa Institute for Theoretical Physics, Kyoto University, Kitashirakawa Oiwakecho, Sakyo-ku, Kyoto 606-8502, Japan

The Hakubi Center for Advanced Research, Kyoto University, Yoshida Ushinomiyacho, Sakyo-ku, Kyoto 606-8501, Japan

E-mail: tomonori.ugajin@yukawa.kyoto-u.ac.jp

ABSTRACT: We holographically compute the Rényi relative divergence $D_{\alpha}\left(\rho_{+} \| \rho_{-}\right)$between two density matrices $\rho_{+}, \rho_{-}$prepared by path integrals with constant background fields $\lambda_{ \pm}$ coupled to a marginal operator in JT gravity. Our calculation is non-perturbative in the difference between two sources $\lambda_{+}-\lambda_{-}$. When this difference is large, the bulk geometry becomes a black hole with the maximal temperature allowed by the Rényi index $\alpha$. In this limit, we find an analytical expression of the Rényi relative divergence, which is given by the on shell action of the back reacted black hole plus the contribution coming from the discontinuous change of the background field.

KEYwords: 2D Gravity, Gauge-gravity correspondence, Holography and condensed matter physics (AdS/CMT)

ArXiv EPrint: 2011.05539 


\section{Contents}

1 Introduction 1

2 Set up 3

3 The holographic set up $\quad 4$

3.1 The construction of the bulk configuration 5

3.1.1 The Janus solution 5

3.1.2 Mapping the solution to Poincare AdS 6

4 On shell reparametrization mode $\quad 6$

4.1 Fixing the temperature 8

$\begin{array}{lll}4.2 & \text { The large source limit } & 9\end{array}$

5 Evaluating the semi-classical action $\quad 9$

5.1 Normal part 9

$\begin{array}{lll}5.2 \text { Defect part } & 10\end{array}$

$\begin{array}{ll}5.3 \text { Matter part } & 10\end{array}$

5.4 The net result 11

6 The calculation of the Rényi relative divergence 11

7 A perturbative expansion of the Rényi relative divergence 12

8 Conclusion and discussions 13

\section{Introduction}

Distance measures on a space of density matrices play a crucial role in quantum information theory. Among them, relative entropy [1] has nice properties such as positivity and monotonicity, which have been efficiently used to prove many intriguing results in quantum field theory, see, for example [2-4]. The relative entropy $S(\rho \| \sigma)$ between two density matrices $\rho, \sigma$ is defined by

$$
S(\rho \| \sigma)=\operatorname{tr} \rho \log \rho-\operatorname{tr} \rho \log \sigma .
$$

Properties of relative entropy are reviewed in [5].

Although it is difficult to explicitly calculate relative entropy in QFT, we can perturvatively compute $S\left(\rho_{0}+\delta \rho \| \rho_{0}\right)$ by expanding it in $\delta \rho$, when the two density matrices are sufficiently close to each other $|\delta \rho| \ll 1$. A nice way to do this is to use the modular flow of the reference state $\rho_{0}[6]$. When this prescription is applied to conformal field theory, it 
turned out the second-order term of the expansion (Fisher information) can be rewritten holographically in terms of the canonical energy on the dual anti de Sitter space $[7,8]$. The result matches with the prediction of the Ryu Takayanagi formula and its covariant generalization [9-11]. See the related discussions [12-21].

Rényi relative divergence (RRD) is a one parameter generalization of relative entropy. It is defined by [22],

$$
D_{\alpha}(\rho \| \sigma)=-\frac{1}{1-\alpha} \log \operatorname{tr}\left[\rho^{\alpha} \sigma^{1-\alpha}\right] .
$$

It has also nice properties which are inherited from relative entropy [23]. Recent discussions of applications of RRD include [24-28]. One of the difficulties in computing RRD is that this quantity breaks the $\mathrm{U}(1)$ translational symmetry of Euclidean time direction. In [29], an efficient way to perturbatively expand RRD by utilizing the resolvent of density matrix was found. In the same paper, it turned out that the second-order term of the expansion of RRD again has a holographic expression. Other studies of perturbative expansion of RRD include [30-32].

These perturbative expansions of relative entropy as well as RRD allow us to efficiently compute them. However, it turns out that in general these series do not converge, as is often the case in perturbations in quantum field theory. This is because $\delta \rho$ is not a bounded operator. Therefore, finding a prescription to resum these series is important. This is also interesting from the holographic point of view, as the higher order terms of the expansions are related to the emergence of full bulk gravitational equations of motion beyond the second order. See also [33] for a recent progress to resolve the non-convergence problem.

As a first step toward this direction, in this paper we holographically compute Renyi relative divergences without using perturbative expansion, in two dimensional JT gravity $[34,35]$ plus a bulk matter theory. We hope this example will give a hint on how to resum the perturbative series found in our previous papers [14, 29]. JT gravity describes the near-horizon dynamics of near extremal black holes [36]. The dynamical degrees of freedom of the theory are the reparametrization modes, which live on the boundary of $A d S_{2}$, break the asymptotic symmetry of the spacetime [37]. The Lagrangian of the resulting effective theory on the boundary is given by the Schwarzian derivative of the reparametrization modes. A nice review of this topic is [38].

We prepare two density matrices by turning on constant background fields coupled to a marginal operator in the boundary theory. We then calculate the RRD between them, which has a path integral expression with a time dependent background field. To calculate this RRD holographically, we first find the bulk configuration whose boundary condition matches with the time-dependent source in the field theory side. This bulk configuration, known as Janus solution was obtained in [39]. In this paper, we will fully use this result. ${ }^{1}$ A nice thing about the solution in two dimensions is that the simplicity of the theory allows us to treat the effect of back reaction exactly for the general Rényi index $\alpha$. In higher dimensions, what we can do best is to work in the regimes where the effects of the back-reaction is small, and the perturbative expansion of the on-shell action gives a good approximation.

\footnotetext{
${ }^{1}$ This solution shares similar properties with its higher dimensional counter part [40, 41].
} 
This paper is organized as follows. In section 2 we define the Rényi relative divergence of our interest in Schwarzian theory coupled to a marginal operator. In addition, we explain the path integral expression of the RRD in this theory. In section 3 we discuss the holographic dual of this setup, ie JT gravity in $A d S_{2}$ coupled to a bulk massless scalar field. We then present the relevant solution, whose on-shell action computes the Rényi relative divergence holographically. In section 4 , we derive the reparametrization mode of the boundary theory which dominates the path integral, from the bulk configuration derived in the previous section, and carefully evaluate its Schwarzian action in section 5. We then assemble these calculations and present the main result in section 6 . In section 7 , we discuss the perturbative expansion of the main result.

\section{Set up}

We study a $0+1$ dimensional theory of reparametrization modes $f(u) \in \operatorname{Diff}\left[S^{1}\right] / \operatorname{SL}(2, \mathbb{R})$, on the circle $S^{1}$ with periodicity $2 \pi$. The action of this theory is given by Schwarzian derivative of $f(u)$,

$$
S_{\mathrm{Sch}}[f]=-C \int_{0}^{2 \pi} d u\left[\{f(u), u\}+\frac{f^{\prime 2}}{2}\right], \quad\{f(u), u\}=-\frac{1}{2}\left(\frac{f^{\prime \prime}}{f^{\prime}}\right)^{2}+\left(\frac{f^{\prime \prime}}{f^{\prime}}\right)^{\prime} .
$$

Throughout this paper, $u$ is the coordinate of $S^{1}$ on which the theory is defined. This action naturally appears as the low energy effective theory of the SYK model [42].

We couple these modes to a scalar operator $\mathcal{O}$. For simplicity, we assume the operator is marginal, ie, its conformal dimension $\Delta$ is 1 , and it is a generalized free field, which means that its correlation functions can be computed by Wick contractions. In this theory, there is a class of density matrices $\left\{\rho_{\lambda}\right\}$, prepared by turning on a constant background field $\lambda \in \mathbb{R}$ for the marginal operator $\mathcal{O}$ on the thermal ensemble $e^{-2 \pi H}$,

$$
\rho_{\lambda}=\exp \left[-2 \pi H+\lambda \int_{0}^{2 \pi} d u \mathcal{O}(u)\right],
$$

where $H$ is Hamiltonian of Schwarzian theory. We will take into account the normalization of the density matrix later.

We would like to compute the Rényi relative divergence $D_{\alpha}\left(\rho_{\lambda_{+}} \| \rho_{\lambda_{-}}\right)$between two such density matrices $\rho_{\lambda_{+}}$and $\rho_{\lambda_{-}}$. To this end, we first calculate the following quantity,

$$
\operatorname{tr} \rho_{\lambda+}^{\alpha} \rho_{\lambda_{-}}^{1-\alpha}=\operatorname{tr}\left[\exp \left(-H+\lambda_{+} \int_{0}^{2 \pi \alpha} \mathcal{O}(u) d u+\lambda_{-} \int_{2 \pi \alpha}^{2 \pi} \mathcal{O}(u) d u\right)\right] .
$$

The right hand side has a path integral expression,

$$
\begin{aligned}
\operatorname{tr} \rho_{\lambda+}^{\alpha} \rho_{\lambda_{-}}^{1-\alpha} & =\int D f \exp \left[-S_{\text {Sch }}[f]-\frac{1}{8 \pi} \int_{0}^{2 \pi} d u_{1} d u_{2} \lambda\left(u_{1}\right) \lambda\left(u_{2}\right)\left\langle\mathcal{O}\left(f\left(u_{1}\right)\right) \mathcal{O}\left(f\left(u_{2}\right)\right)\right\rangle\right] \\
& =\int D f \exp \left[-S_{\operatorname{Sch}}[f]-\frac{1}{8 \pi} \int_{0}^{2 \pi} d u_{1} d u_{2} \lambda\left(u_{1}\right) \lambda\left(u_{2}\right)\left(\frac{f^{\prime}\left(u_{1}\right) f^{\prime}\left(u_{2}\right)}{\sin \left(\frac{f\left(u_{1}\right)-f\left(u_{2}\right)}{2}\right)^{2}}\right)\right],
\end{aligned}
$$




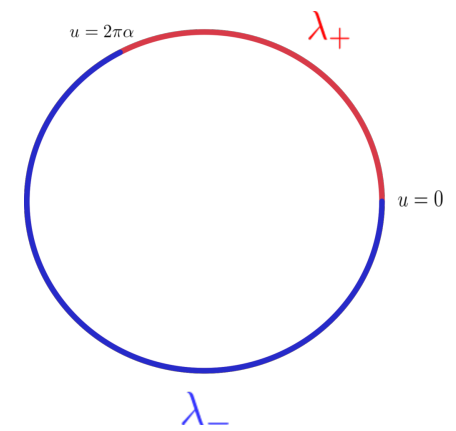

Figure 1. The background field profile $\lambda(u)$ for the marginal operator which appears in the path integral. The source jumps discontinuously at $u=0$ and $u=2 \pi \alpha$.

with the time-dependent background field $\lambda(u)$ which is coupled to the marginal scalar operator $\mathcal{O}$,

$$
\lambda(u)= \begin{cases}\lambda_{+} & 0<u<2 \pi \alpha \\ \lambda_{-} & 2 \pi \alpha<u<2 \pi,\end{cases}
$$

see figure 1. $\left\langle\mathcal{O}\left(f\left(u_{1}\right)\right) \mathcal{O}\left(f\left(u_{2}\right)\right)\right\rangle$ in the second line of $(2.4)$ is the two point function of the marginal operator $\mathcal{O}$ in the presence of the reparametrization mode. As we will see in the next section, this action is related holographically to JT gravity coupled to a massless free scalar field theory in $A d S_{2}$. We evaluate this path integral in the semiclassical limit $C \gg 1$.

\section{The holographic set up}

The setup we are considering has a gravity dual, namely $A d S_{2}$ JT gravity coupled to a free massless scalar field $\chi$ which is dual to the marginal operator $\mathcal{O}$. The total bulk action is

$$
I=-\frac{\phi_{0}}{16 \pi G}\left[\int d x^{2} \sqrt{g} R+2 \int K\right]-\frac{1}{16 \pi G}\left[\int d x^{2} \sqrt{g} \Phi(R+2)+2 \int \phi_{b} K\right]+I_{M}[g, \chi],
$$

JT gravity part involves dilaton $\Phi$ as well as metric $g_{\mu \nu}$. The matter part $I_{M}[g, \chi]$ is the action of free scalar field $\chi$,

$$
I_{M}=-\frac{1}{2} \int d x^{2} \sqrt{g}\left(g^{a b} \nabla_{a} \chi \nabla_{b} \chi-m^{2} \chi^{2}\right) .
$$

One notable feature of the bulk action is that there is no coupling between the dilaton $\phi$ and the matter field $\chi$. Hereafter, we set the mass of the scalar field to zero $m=0$, so that the primary $\mathcal{O}$ is a marginal operator $\Delta_{\mathcal{O}}=1$.

This action describes excitations on top of an higher dimensional extremal black hole geometry, dimensionally reduced to two dimensions. The first term of the action (3.1) is purely topological, describes the grand state degeneracy of the extremal black hole. Therefore, the constant $\phi_{0}$ corresponds to the entropy of the higher dimensional extremal black hole. The second term of the action (3.1) captures excitations on top of the extremal black hole, and the total dilaton $\Phi+\phi_{0}$ corresponds to the entropy of the excited black 
hole. In order for the effective description valid, we need $\phi_{0} \gg \Phi$. The equations of motion for dilaton $\Phi$ and the metric $g_{\mu \nu}$ is

$$
\nabla_{\mu} \nabla_{\nu} \Phi-g_{\mu \nu} \nabla^{2} \Phi-g_{\mu \nu} \Phi=8 \pi G T_{\mu \nu}[\chi], \quad R+2=0
$$

where the stress tensor $T_{\mu \nu}[\chi]$ of the massless scalar field $\chi$,

$$
T_{\mu \nu}[\chi]=\nabla_{\mu} \chi \nabla_{\nu} \chi-\frac{g_{\mu \nu}}{2}\left(\nabla^{\rho} \chi \nabla_{\rho} \chi\right) .
$$

The second equation of (3.3) fixes the metric to that of pure $A d S_{2}$. In Poincare coordinates, this metric is given by

$$
d s^{2}=\frac{d t^{2}+d z^{2}}{z^{2}}
$$

Due to the equations of motion (3.3), the bulk action (3.1) is reduced to the field theory action (2.3) on $S^{1}$ [37], once we fix the location of the boundary $(t, z)=(t(u), z(u))$ in the bulk, and the relation between $t(u)$ and the reparametrization mode $f(u)$, which will be discussed later. In doing so, we also need the identification of the coupling constants on both sides,

$$
C=\frac{\bar{\phi}}{8 \pi G}
$$

$t(u)$ and $z(u)$ define an embedding of the boundary $S^{1}$ to Poincare $A d S_{2}$. Furthermore, the symmetry imposes the relation $z(u)=\varepsilon t^{\prime}(u)$, where $\varepsilon$ is the UV cut-off of the boundary theory. The location of the boundary line $t(u)$ is specified by imposing the boundary conditions

$$
\Phi\left(t(u), \varepsilon t^{\prime}(u)\right)=\frac{\bar{\phi}}{\varepsilon}, \quad \chi\left(t(u), \varepsilon t^{\prime}(u)\right)=\lambda(u) .
$$

In this way, the task of computing Rényi relative divergence in the semiclassical limit $C \gg 1$ is reduced to find the corresponding solution of the gravitational equations motion (3.3), and specifying the reparametrization mode $f(u)$ from the solution.

\subsection{The construction of the bulk configuration}

\subsubsection{The Janus solution}

Our starting point is the Janus solution, obtained in [39]. This solution also naturally appears in a recent discussion on the entanglement entropy between two disjoint universes and its relation to island formula [43]. Also, this class of solutions has been also discussed in slightly different contexts $[44,45]$. In the global AdS coordinates $\left(\mu, t_{s}\right)$ where the metric takes the following form,

$$
d s^{2}=\frac{d t_{s}^{2}+d \mu^{2}}{\cos ^{2} \mu}, \quad-\frac{\pi}{2}<\mu<\frac{\pi}{2}
$$

this solution is given by

$$
\Phi\left(\mu, t_{s}\right)=\Phi_{0}\left(\mu, t_{s}\right)-4 \pi G \gamma^{2}(1+\mu \tan \mu),
$$


and

$$
\chi(\mu)=\gamma\left(\mu-\frac{\pi}{2}\right)+\lambda_{+},
$$

where $\gamma$ is the difference between the background fields, $\gamma=\left(\lambda_{-}-\lambda_{+}\right) / \pi$, which is necessary to impose the boundary condition,

$$
\left.\chi\right|_{\mu=\frac{\pi}{2}}=\lambda_{+},\left.\quad \chi\right|_{\mu=-\frac{\pi}{2}}=\lambda_{-}
$$

$\Phi_{0}\left(\mu, t_{s}\right)$ is the "souceless" of the dilaton, satisfying the equations of motion (3.3) with $T_{\mu \nu}[\chi]=0$. This part will be fixed later.

\subsubsection{Mapping the solution to Poincare AdS}

To relate the dilaton profile (3.9) to the reparametrization mode $f(u)$, it is convenient to write down the solution in Poincare coordinates with the metric (3.5).

The relation between global and Poincare coordinates is

$$
z+i t=e^{i \mu+t_{s}} \leftrightarrow t_{s}=\frac{1}{2} \log \left[z^{2}+t^{2}\right], \quad \mu=\tan ^{-1} \frac{t}{z} .
$$

The dilaton profile in Poincare coordinates is,

$$
\Phi(z, t)=\Phi_{0}(z, t)-4 \pi G \gamma^{2}\left(1+\frac{t}{z} \tan ^{-1} \frac{t}{z}\right) .
$$

the sourceless part of dilaton $\Phi_{0}$ is in general,

$$
\Phi_{0}(z, t)=\frac{\xi\left(t^{2}+z^{2}\right)+\kappa t+\eta}{z} .
$$

Finally, the scalar field $\chi$ profile is

$$
\chi(t, z)=\gamma\left(\tan ^{-1} \frac{t}{z}-\frac{\pi}{2}\right)+\lambda_{+} .
$$

Its boundary value depends on the sign of the time-like coordinate $t$,

$$
\chi(t, z) \rightarrow\left\{\begin{array}{ll}
\lambda_{+} & t>0 \\
\lambda_{-} & t<0
\end{array}, z \rightarrow 0 .\right.
$$

\section{On shell reparametrization mode}

Having specified the solution of the equations of motion (3.13) and (3.15), now the task is to find the location of the boundary $(t, z)=\left(t(u), \varepsilon t^{\prime}(u)\right)$ on which the boundary conditions (3.7) are satisfied. This also fixes the parameters $(\xi, \kappa, \eta)$ of the sourceless part of dilaton $\Phi_{0}(3.14)$. At the same time we relate the boundary location $t(u)$ in Poincare $\mathrm{AdS}_{2}$ to the boundary location in Poincare disc, which is identified with the reparametrization mode $f(u)$ in the Schwarzian action (2.1), via an appropriate bulk diffeomorphism. 
The boundary location for sourceless dilaton. We begin our discussion from the case where the dilaton is sourceless $\Phi=\Phi_{0}$, ie when the stress tensor of the scalar field vanishes $T_{\mu \nu}[\chi]=0$. The general form of such dilaton profile is given by (3.14). We want an embedding $t(u)$, which maps the segment $I^{+}: 0<u<2 \pi \alpha$ (the red interval in figure 1) on the boundary circle of Poincare disc $S^{1}$, to $t>0$ part of the boundary of Poincare $\mathrm{AdS}_{2}$, and its complement $I^{-}: 2 \pi \alpha<u<2 \pi$ (the blue interval in figure 1) to $t<0$. Such a map is given by

$$
t(u)=-\frac{\sin \frac{u}{2}}{\sin \left(\frac{u-2 \pi \alpha}{2}\right)} .
$$

The sourceless dilaton profile which satisfies the boundary condition (3.7), is given by choosing the coefficients $(\xi, \kappa, \eta)$ in $(3.14)$,

$$
\xi=\frac{\bar{\phi}}{\sin \pi \alpha}, \quad \kappa=\frac{2 \bar{\phi}}{\tan \pi \alpha}, \quad \eta=\frac{\bar{\phi}}{\sin \pi \alpha} .
$$

Note that since the Schwarzian derivative of (4.1) is still constant

$$
\{t(u), u\}=\frac{1}{2},
$$

the introduction of the index $\alpha$ does not change the thermodynamic quantities of the black hole as it should be.

The boundary location in the presence of the source. Now let us turn on the source $\gamma$. We find that by choosing the following coefficients $(\xi, \kappa, \eta)$ in $(3.14)$, one can satisfy the boundary condition.

$$
\xi=\frac{\bar{\phi} \nu}{\sin \pi \alpha \nu}, \quad \kappa=\frac{2 \bar{\phi} \nu}{\tan \pi \alpha \nu}+2 \pi^{2} G \gamma^{2}, \quad \eta=\frac{\bar{\phi} \nu}{\sin \pi \alpha \nu} .
$$

Here we introduce $\nu$, which will be identified with the temperature of the backreacted black hole, caused by the source $\gamma$.

We should remark that our choice of the sourceless part $\Phi_{0}$ is slightly different from the choice of [39]. In [39], $\Phi_{0}$ was fixed by demanding that the temperature $\nu$ of the back reacted black hole is always fixed, ie independent of $\gamma$. This is not convenient for our purpose, since with this choice the boundary conditions (3.7) can not be satisfied. Indeed, we will see later that onece we choose $\Phi_{0}$ which satisfies the boundary condition, then the temperature of the backreacted black hole does depend on $\gamma$.

Let us first consider the interval $0<u<2 \pi \alpha$. If we choose the map,

$$
t(u)=-\frac{\sin \frac{\nu u}{2}}{\sin \left(\frac{\nu u-2 \pi \alpha \nu}{2}\right)}
$$

then $I^{+}$is correctly mapped to $t>0$, and satisfies the boundary condition for the scalar field $\chi(u)=\lambda_{+}$. Furthermore, due to the choice of the sourceless part,the pull back of the total dilaton on $I^{+}$also satisfies the boundary condition $\Phi\left(t(u), \varepsilon t^{\prime}(u)\right)=\bar{\phi} / \varepsilon$. 
On the complement $I^{-}: 2 \pi \alpha<u<2 \pi$, we employ a generalized ansatz for $t(u)$,

$$
t(u)=-\frac{\sin \frac{f(u)}{2}}{\sin \left(\frac{f(u)-2 \pi \nu \alpha}{2}\right)} .
$$

In addition, for the continuity we demand $f(2 \pi \alpha)=2 \pi \alpha \nu$. Then the pullback of the dilaton profile (3.13) with (4.4) on this curve is

$$
\Phi(u) \equiv \varepsilon \Phi\left(t(u), \varepsilon t^{\prime}(u)\right)=\frac{\phi_{0}}{f^{\prime}}-4 \pi G \gamma^{2}\left[-\frac{\pi}{2}+\tan ^{-1}\left(\frac{h(u)}{\varepsilon f^{\prime}}\right)\right]\left(\frac{h(u)}{\varepsilon f^{\prime}}\right),
$$

with

$$
h(u)=\frac{-2}{\sin (\pi \nu \alpha)} \sin \frac{f}{2} \sin \left(\frac{f-2 \pi \nu \alpha}{2}\right) .
$$

When $|h(u)| \gg \varepsilon f^{\prime}(u)$, we can replace arectan in (4.7) with either $\frac{\pi}{2}$ or $-\frac{\pi}{2}$, depending on the sign of $h(u)$. For example we can do this on the following regions $I_{\varepsilon}^{+}: \sqrt{\varepsilon}<u<2 \pi \alpha-\sqrt{\varepsilon}$ and $I_{\varepsilon}^{-}: 2 \pi \alpha+\sqrt{\varepsilon}<u<2 \pi-\sqrt{\varepsilon}$, where $h(u)$ is sufficiently large, in the $\varepsilon \rightarrow 0$ limit.

If we impose the boundary condition in (3.7) for the dilaton, we get

$$
f^{\prime}(u)=\left\{\begin{array}{l}
\nu, \quad u \in I_{\varepsilon}^{+}, \\
\nu-\frac{8 \pi^{2} \gamma^{2} G}{\phi \sin (\pi \nu \alpha)} \sin \frac{f}{2} \sin \left(\frac{f-2 \pi \nu \alpha}{2}\right) \quad u \in I_{\varepsilon}^{-} .
\end{array}\right.
$$

\subsection{Fixing the temperature}

Now let us fix the temperature $\nu$ of the backreacted black hole. To do this, recall the expression two-point function of the marginal operator $\mathcal{O}$ dual to the bulk scalar field $\chi$,

$$
\left\langle\mathcal{O}\left(u_{1}\right) \mathcal{O}\left(u_{2}\right)\right\rangle=\frac{f^{\prime}\left(u_{1}\right) f^{\prime}\left(u_{2}\right)}{4 \sin ^{2}\left(\frac{f\left(u_{1}\right)-f\left(u_{2}\right)}{2}\right)} .
$$

In order for the correlator to have the appropriate periodicity $u_{1} \sim u_{1}+2 \pi$, the reparametrization mode $f(u)$ has to satisfy $f(2 \pi)=2 \pi$. From (4.9), we see that this condition is equivalent to

$$
\int_{2 \pi \alpha \nu}^{2 \pi} \frac{d f}{\left[\nu-\frac{8 \pi^{2} \gamma^{2} G}{\phi \sin (\pi \nu \alpha)} \sin \frac{f}{2} \sin \left(\frac{f-2 \pi \nu \alpha}{2}\right)\right]}=2 \pi(1-\alpha) .
$$

This equation determines the temperature $\nu$ of the back reacted black hole, as a function of $\gamma$ and $\alpha$. In general, it is difficult to obtain its explicit solution.However, one can perturbatively solve this equation around $\gamma=0$, let $\nu=1+\delta \nu$, then,

$$
\delta \nu=\frac{2 \pi \gamma^{2} G}{\bar{\phi} \sin (\pi \alpha)}[2 \pi(1-\alpha) \cos \pi \alpha+2 \sin \pi \alpha]+O\left(\gamma^{4}\right) .
$$




\subsection{The large source limit}

When the difference of the source $\gamma$ is large, we can also solve this equation exactly. In this limit, the temperature of the back reacted black hole gets maximal, ie $\nu \rightarrow 1 / \alpha$. If the temperature could be larger than this value, we were unable to impose the periodicity condition $f(2 \pi)=2 \pi$. Indeed, when $\gamma \gg 1$ one can consistently solve the equation for $\nu$ by $1 / \gamma^{2}$ expansion.

Since $\nu \rightarrow 1 / \alpha$, the range of the integration (4.11), become smaller, $2 \pi \alpha \nu \rightarrow 2 \pi$. In order for the integral non zero, the integrand has to diverge in this limit. In particular, by expanding the integrand near $f \sim 2 \pi$, we see that in order for this to happen, $\nu$ has to satisfy

$$
\nu=\frac{1}{\alpha}-\frac{\bar{\phi}}{2 \alpha \pi^{3} G \gamma^{2}}+\cdots .
$$

We emphasize that this reasoning is only valid when $\alpha \neq 1$ or equivalently, when the left hand side of (4.11) is non-zero. In particular, when we calculate the relative entropy,

$$
S\left(\rho_{+} \| \rho_{-}\right)=\lim _{\alpha \rightarrow 1} D_{\alpha}\left(\rho_{+} \| \rho_{-}\right),
$$

we can not use the expansion, since this limit involves $\alpha \sim 1$.

\section{Evaluating the semi-classical action}

Having specified the reparametrization mode which dominates the path integral (2.3), in this section, we evaluate its on shell action. It is straightforward to evaluate the action on $I_{\varepsilon}^{ \pm}$where the reparametrization mode is given by (4.9). However near $u=02 \pi \alpha$, lets say $C_{\varepsilon}^{+}:-\sqrt{\varepsilon}<u<+\sqrt{\varepsilon}$, and $C_{\varepsilon}^{-}: 2 \pi \alpha-\sqrt{\varepsilon}<u<2 \pi \alpha+\sqrt{\varepsilon}$, the boundary source $\lambda(u)$ changes discontinuously, and total dilaton profile behaves like a step function. Accordingly, higher order derivatives of the reparametrization mode develop delta functional singularities. Therefore, in evaluating its Schwarzian action $S_{\text {Sch }}$, we need special care. It is convenient to split the integral into four pieces.

$$
\begin{aligned}
S_{\mathrm{Sch}} & =-C \lim _{\varepsilon \rightarrow 0}\left[\int_{C_{\varepsilon}^{+}}+\int_{I_{\varepsilon}^{+}}+\int_{C_{\varepsilon}^{-}}+\int_{I_{\varepsilon}^{-}}\right] d u\left[\frac{f^{\prime 2}}{2}-\frac{1}{2}\left(\frac{f^{\prime \prime}}{f^{\prime}}\right)^{2}+\left(\frac{f^{\prime \prime}}{f^{\prime}}\right)^{\prime}\right] \\
& \equiv \lim _{\varepsilon \rightarrow 0} S_{C_{\varepsilon}^{+}}+S_{I_{\varepsilon}^{+}}+S_{C_{\varepsilon}^{-}}+S_{I_{\varepsilon}^{-}} .
\end{aligned}
$$

\subsection{Normal part}

It is straightforward to evaluate $S_{I_{\varepsilon}^{+}}$and $S_{I_{\varepsilon}^{-}}$. This is because the expression of the reparametrization mode gets simplified in these regions (4.9). In general, when a reparametrization mode satisfies the relation,

$$
f^{\prime}=a-b \cos (f-\delta), \quad \delta \in \mathbb{R},
$$

then, the Lagrangian is constant,

$$
L_{\mathrm{Sch}}[f]=\frac{f^{\prime 2}}{2}-\frac{1}{2}\left(\frac{f^{\prime \prime}}{f^{\prime}}\right)^{2}+\left(\frac{f^{\prime \prime}}{f^{\prime}}\right)^{\prime}=\frac{a^{2}-b^{2}}{2} .
$$


For the remparametrization mode of interest (4.9), we obtain

$$
L_{\mathrm{Sch}}[f]=\left\{\begin{array}{l}
\frac{\nu^{2}}{2}, \quad u \in I_{\varepsilon}^{+} \\
\frac{1}{2}\left(\nu-\frac{4 \pi^{2} G \gamma^{2}}{\bar{\phi} \tan \pi \alpha \nu}\right)^{2}-\frac{1}{2}\left(\frac{4 \pi^{2} G \gamma^{2}}{\bar{\phi} \sin \pi \alpha \nu}\right)^{2}, \quad u \in I_{\varepsilon}^{-}
\end{array}\right.
$$

$L_{\mathrm{Sch}}$ is related to the stress energy expectation value $\left\langle T_{00}\right\rangle$ of the boundary theory [37]

$$
\left\langle T_{00}\right\rangle=C L_{\mathrm{Sch}}[f] .
$$

Therefore the expression (5.5) implies, when the dilaton profile is analytically continued to Lorenzian signature, it represents a two sided black hole, in which the temperatures of the left and right horizons are different. However, in the large $\gamma$ limit, we can see that

$$
\frac{1}{2}\left(\nu-\frac{4 \pi^{2} G \gamma^{2}}{\bar{\phi} \tan \pi \alpha \nu}\right)^{2}-\frac{1}{2}\left(\frac{4 \pi^{2} G \gamma^{2}}{\bar{\phi} \sin \pi \alpha \nu}\right)^{2} \rightarrow \frac{\nu^{2}}{2},
$$

by plugging (4.13). This indicates that two black hole temperatures coincide in this limit.

By combining these results, the normal part of the action is given by

$$
\lim _{\varepsilon \rightarrow 0} S_{I_{\varepsilon}^{+}}+S_{I_{\varepsilon}^{-}}=-\frac{\bar{\phi}}{8 \pi G}\left\{\pi \alpha \nu^{2}+\pi(1-\alpha)\left[\left(\nu-\frac{4 \pi^{2} G \gamma^{2}}{\bar{\phi} \tan \pi \alpha \nu}\right)^{2}-\left(\frac{4 \pi^{2} G \gamma^{2}}{\bar{\phi} \sin \pi \alpha \nu}\right)^{2}\right]\right\} .
$$

\subsection{Defect part}

Now let us evaluate the action on the transition regions $C_{\varepsilon}^{+}, C_{\varepsilon}^{-}$. Naively speaking, since we take $\varepsilon \rightarrow 0, S_{C_{\varepsilon}^{+}} S_{C_{\varepsilon}^{-}}$are vanishing. However, we see that since the last term of the Lagrangian (5.1) is highly singular due to the discontinuity of the source profile $\lambda(u)$, and $S_{C_{\varepsilon}^{+}} S_{C_{\varepsilon}^{-}}$are indeed non vanishing even in the $\varepsilon \rightarrow 0$ limit. Indeed the sum of these two terms are given by

$$
\begin{aligned}
\lim _{\varepsilon \rightarrow 0} S_{C_{\varepsilon}^{+}}+S_{C_{\varepsilon}^{-}} & =-C \lim _{\varepsilon \rightarrow 0}\left[\left(\frac{f^{\prime \prime}}{f^{\prime}}\right)_{u=\sqrt{\varepsilon}}-\left(\frac{f^{\prime \prime}}{f^{\prime}}\right)_{2 \pi-\sqrt{\varepsilon}}\right]-C \lim _{\varepsilon \rightarrow 0}\left[\left(\frac{f^{\prime \prime}}{f^{\prime}}\right)_{u=\sqrt{\varepsilon}}-\left(\frac{f^{\prime \prime}}{f^{\prime}}\right)_{2 \pi-\sqrt{\varepsilon}}\right] \\
& =-\pi \gamma^{2} .
\end{aligned}
$$

Here, we used the fact that the integral of the first two terms of the Lagrangian vanishes in the $\varepsilon \rightarrow 0$ limit. Two terms of the right hand side of above equation can be evaluated by using the approximate expressions (4.9) for $f(u)$.

\subsection{Matter part}

Finally, we need to evaluate the matter action related to the marginal operator $\mathcal{O}$, which explicitly depends on the time dependent source $\lambda(u)(2.5)$.

$$
-I_{M}=\frac{1}{8 \pi} \int_{0}^{2 \pi} d u_{1} \int_{0}^{2 \pi} d u_{2} \lambda\left(u_{1}\right) \lambda\left(u_{2}\right) \frac{f^{\prime}\left(u_{1}\right) f^{\prime}\left(u_{2}\right)}{\sin ^{2}\left(\frac{f\left(u_{1}\right)-f\left(u_{2}\right)}{2}\right)} .
$$


In evaluating this integral, we have to properly regulate the singularity at $u_{1}=u_{2}$. This regularization can be implemented by introducing a UV cutoff $\varepsilon$ to the integral as

$$
\begin{aligned}
-I_{M} & =\frac{1}{8 \pi} \int_{\varepsilon}^{2 \pi-\varepsilon} d u_{1}\left[\int_{u_{1}+\varepsilon}^{2 \pi}+\int_{0}^{u_{1}-\varepsilon}\right] d u_{2} \lambda\left(u_{1}\right) \lambda\left(u_{2}\right) \frac{f^{\prime}\left(u_{1}\right) f^{\prime}\left(u_{2}\right)}{\sin ^{2}\left(\frac{f\left(u_{1}\right)-f\left(u_{2}\right)}{2}\right)} \\
& =\frac{2 \lambda_{+}^{2} \alpha}{\varepsilon}+\frac{2 \lambda_{-}^{2}(1-\alpha)}{\varepsilon}-\pi \gamma^{2} \log \left|\frac{\sin \pi \alpha \nu}{\varepsilon}\right|,
\end{aligned}
$$

here, we used the definition $\gamma=\left(\lambda_{-}-\lambda_{+}\right) / 2 \pi$.

\subsection{The net result}

By combining these results, The total action is given by

$$
\begin{aligned}
\log \rho_{+}^{\alpha} \rho_{-}^{1-\alpha} & =-\lim _{\varepsilon \rightarrow 0}\left[S_{I_{\varepsilon}^{+}}+S_{I_{\varepsilon}^{-}}+S_{C_{\varepsilon}^{+}}+S_{C_{\varepsilon}^{-}}\right]-I_{M} \\
& =\frac{\bar{\phi}}{8 \pi G}\left\{\pi \alpha \nu^{2}+\pi(1-\alpha)\left[\left(\nu-\frac{4 \pi^{2} G \gamma^{2}}{\bar{\phi} \tan \pi \alpha \nu}\right)^{2}-\left(\frac{4 \pi^{2} G \gamma^{2}}{\bar{\phi} \sin \pi \alpha \nu}\right)^{2}\right]\right\}+\pi \gamma^{2} \\
& +\frac{2 \lambda_{+}^{2} \alpha}{\varepsilon}+\frac{2 \lambda_{-}^{2}(1-\alpha)}{\varepsilon}-\pi \gamma^{2} \log \left|\frac{\sin \pi \alpha \nu}{\varepsilon}\right|
\end{aligned}
$$

\section{The calculation of the Rényi relative divergence}

Now let us assemble the results so far, to calculate the Rényi relative divergence $D_{\alpha}\left(\rho_{+} \| \rho_{-}\right)$ of our interest. Including the normalization, this quantity is defined by

$$
D_{\alpha}\left(\rho_{+} \| \rho_{-}\right)=-\frac{1}{1-\alpha} \log \left[\frac{\operatorname{tr}\left[\rho_{+}^{\alpha} \rho_{-}^{1-\alpha}\right]}{\left(\operatorname{tr}\left[\rho_{+}\right]\right)^{\alpha}\left(\operatorname{tr}\left[\rho_{-}\right]\right)^{1-\alpha}}\right] .
$$

The denominator is computed by evaluating the on-shell action in the presence of the constant scalar field $\chi=\lambda_{ \pm}$,

$$
\log \operatorname{tr} \rho_{+}=\frac{\bar{\phi}}{8 G}+\frac{2 \lambda_{+}^{2}}{\varepsilon}, \quad \log \operatorname{tr} \rho_{-}=\frac{\bar{\phi}}{8 G}+\frac{2 \lambda_{-}^{2}}{\varepsilon} .
$$

By combining this with (5.12), we arrive

$$
\begin{aligned}
D_{\alpha}\left(\rho_{+} \| \rho_{-}\right)= & -\frac{\bar{\phi}}{8 G(1-\alpha)}\left\{\alpha \nu^{2}+(1-\alpha)\left[\left(\nu-\frac{4 \pi^{2} G \gamma^{2}}{\bar{\phi} \tan \pi \alpha \nu}\right)^{2}-\left(\frac{4 \pi^{2} G \gamma^{2}}{\bar{\phi} \sin \pi \alpha \nu}\right)^{2}\right]-1\right\} \\
& +\frac{\pi \gamma^{2}}{1-\alpha}\left(-1+\log \left|\frac{\sin \pi \alpha \nu}{\varepsilon}\right|\right)
\end{aligned}
$$

The result involves the temperature of the back reacted black hole $\nu$, which is specified by solving (4.11). In particular, when $\gamma$ is large, it is given by (4.13). From this we obtain an analytic expression of the Rényi relative divergence in this limit $\gamma \gg 1$,

$$
D_{\alpha}\left(\rho_{+}|| \rho_{-}\right)=-\frac{\bar{\phi}}{8 G \alpha^{2}}(1+\alpha)+\frac{\pi \gamma^{2}}{1-\alpha}\left(-1+\log \left|\frac{1}{\varepsilon} \sin \left(\frac{\bar{\phi}}{2 \pi^{2} G \gamma^{2}}\right)\right|\right),
$$


In this expression, $\gamma^{2} \ll 1 / \varepsilon$ is understood also, in order for the validity of the bulk effective theory. We remark that the approximate expression of the black hole temperature (4.13) is not valid near $\alpha=1$. This is the reason $\alpha \rightarrow 1$ limit of (6.4) is not smooth.

In [29], we obtained a general formula for a perturbative expansion of Rényi relative divergence. This was done by writing

$$
\operatorname{tr} \rho^{\alpha} \sigma^{1-\alpha}=\frac{1}{2 \pi i} \int_{C} d z z^{\alpha} \operatorname{tr}\left[\frac{\sigma^{1-\alpha}}{z-\rho}\right]
$$

and expanding the denominator of the right hand side, by assuming $\delta \rho=\rho-\sigma$ is small. In our case, this amounts to expand the RRD with respect to the background field $\lambda$. The result is concisely summarized in terms of integrals along the modular flow of the reference state $\sigma$. However, the expansion obtained in this way is an asymptotic series, so we need to find an prescription to resum the series, to make it well defined. Now, given the exact result (6.3) at hand, it would be interesting to study how the resummation works, and the result obtained in this paper is reproduced from the asymptotic series.

\section{A perturbative expansion of the Rényi relative divergence}

Thus far, we computed the Rényi relative divergence only in the large $\gamma$ limit. This is because we can solve the equation for the temperature of the backreacted black hole $\nu$ only in this limit. Of course, we can study this divergence in another limit, namely in the small $\gamma$ limit, by the perturbative expansion from $\gamma=0$.

The non trivial part of the reparametrization mode can be obtained from

$$
\int_{2 \pi \alpha \lambda}^{f(u)} \frac{d f}{\left[\nu-\frac{8 \pi^{2} \gamma^{2} G}{\bar{\phi} \sin (\pi \lambda \alpha)} \sin \frac{f}{2} \sin \left(\frac{f-2 \pi \lambda \alpha}{2}\right)\right]}=u-2 \pi \alpha .
$$

One can perturbatively solve this equation near $\gamma=0$,

$$
f(u)=u+\gamma^{2} g(u)+O\left(\gamma^{4}\right) .
$$

Accordingly, the on shell Schwarzian action can be expanded like

$$
S_{\text {Sch }}[f]=\sum_{n=0}^{\infty} \gamma^{2 n} S_{2 n}=S_{0}+\gamma^{2} S_{2}+\gamma^{4} S_{4}+O\left(\gamma^{6}\right) .
$$

The leading correction $S_{2}$ is vanishing because of periodicity $g(0)=g(2 \pi)$. Thus, the first nontrivial part of the action starts from $S_{4}$. This gives the leading gravitational contribution to the Rényi relative divergence.

Eq. (7.1) is equivalent to

$$
f^{\prime}(u)=\nu-\frac{8 \pi^{2} \gamma^{2} G}{\bar{\phi} \sin \pi \alpha} \sin \frac{u}{2} \sin \frac{u-2 \pi \alpha}{2}+O\left(\gamma^{4}\right), \quad u \in I_{\varepsilon}^{-} .
$$

By integrating this expression we get,

$$
g(u)=\left[\delta \nu-\frac{4 \pi^{2} \gamma^{2} G}{\bar{\phi} \sin \pi \alpha}((u-2 \pi \alpha) \cos \pi \alpha-(\sin (u-\pi \alpha)-\sin \pi \alpha)],\right.
$$


where $\delta \nu$ is given by (4.12) which we reproduce here,

$$
\delta \nu=\frac{2 \pi \gamma^{2} G}{\bar{\phi}}[2 \pi(1-\alpha) \cot \pi \alpha+2] .
$$

Notice that $g(u)$ satisfies the periodicity condition $g(u)=g(2 \pi)=0$. One can easily expand the Schwarzian action up to $\gamma^{4}$ term,

$$
\begin{aligned}
\gamma^{4} S_{4} & =\int_{0}^{2 \pi} d u\left(g^{\prime}(u)\right)^{2}-\left(g^{\prime \prime}(u)\right)^{2} \\
& =\int_{0}^{2 \pi \alpha} d u\left(g^{\prime}(u)\right)^{2}+\int_{0}^{2 \pi \alpha} d u\left(g^{\prime}(u)\right)^{2}-\left(g^{\prime \prime}(u)\right)^{2} .
\end{aligned}
$$

By plugging (7.5) into the action, we obtain

$$
\gamma^{4} S_{4}=-2 \pi(\delta \nu)^{2}+\frac{8 \pi^{3} \gamma^{2} G}{\bar{\phi}} \delta \nu \cot \pi \alpha
$$

Similarly, we can expand the matter part (5.11) up to $\gamma^{4}$ term,

$$
-I_{M}=\frac{2 \lambda_{+}^{2}}{\varepsilon}-\pi \gamma^{2}\left[\log \left|\frac{\sin \pi \alpha}{\varepsilon}\right|+\pi \alpha \cot \pi \alpha \delta \nu\right] .
$$

By assembling these results, we obtain the perturbative expansion of the Rényi relative divergence (6.1) up to $\gamma^{4}$ order,

$D_{\alpha}\left(\rho_{+}|| \rho_{-}\right)=-\frac{1}{1-\alpha}\left(-2 \pi(\delta \nu)^{2}+\frac{8 \pi^{3} \gamma^{2} G}{\bar{\phi}} \delta \nu \cot \pi \alpha\right)+\frac{\pi \gamma^{2}}{(1-\alpha)}\left[\log \left|\frac{\sin \pi \alpha}{\varepsilon}\right|+\pi \alpha \cot \pi \alpha \delta \nu\right]$.

The relative entropy $S\left(\rho_{+} \| \rho_{-}\right)$is given by the $\alpha \rightarrow 1$ limit of the divergence,

$$
S\left(\rho_{+} \| \rho_{-}\right)=\operatorname{tr} \rho \log \rho-\operatorname{tr} \rho \log \sigma=\lim _{\alpha \rightarrow 1} D_{\alpha}\left(\rho_{+} \| \rho_{-}\right) .
$$

This relative entropy has an additional divergence coming from the derivative of $\log |\sin \pi \alpha|$. After introducing the UV cut off for this, the perturbative expansion of the relative entropy is

$$
S\left(\rho_{+} \| \rho_{-}\right)=\frac{\gamma^{2}}{\varepsilon}-\frac{4 \pi^{4} G \gamma^{4}}{3 \bar{\phi}}-\frac{2 \pi}{3}\left(\frac{4 \pi^{2} \gamma^{2} G}{\bar{\phi}}\right)^{2}+O\left(\gamma^{4}\right)
$$

Positivity of the relative entropy is guaranteed, since the first term is positively divergent.

\section{Conclusion and discussions}

We studied the Rényi relative divergence (RRD) $D_{\alpha}\left(\rho_{+} \| \rho_{-}\right)$between two states prepared by a path integral in the presence of constant back ground fields coupled to a marginal operator $\mathcal{O}$ in $0+1$ dimensional Schwarzian theory. In doing so, we utilized the holographic set up, where JT gravity is coupled to a massless scalar field, dual to the marginal operator $\mathcal{O}$. 
The technical challenge of such a calculation is to deal with the back reaction of the bulk scalar field which is sourced by the time dependent background field on the boundary. Due to this difficulty, the main tool to study this kind of RRD has been the perturbative expansion with respect to the background field. However, the simplicity of JT gravity allows us to fully specify such back reaction in an exact manner. Having this advantage in mind, in the body of this paper, we mainly focused on the regime where the back reaction of the source is significant. The back reaction made the temperature $\nu$ of the black hole maximal, allowed by the Rényi index $\alpha$, ie $\nu \rightarrow \frac{1}{\alpha}$. The resulting RRD was given by the on shell action of this black hole plus the contribution of the discontinuity of the source.

Let us briefly discuss the Lorenzian geometry of the solution (3.9). In global coordinates, the Lorenzian dilaton profile is given by

$$
\Phi=\left(\frac{\nu \bar{\phi}}{\sin \pi \alpha \nu}\right) \frac{\cos \tau}{\cos \mu}+\left(\frac{2 \nu \bar{\phi}}{\tan \pi \alpha \nu}+2 \pi^{2} G \gamma^{2}\right) \tan \mu-4 \pi G \gamma^{2}(\mu \tan \mu+1) .
$$

It represents a two sided black hole with a long interior region. The locations of these horizons are the critical points of the Lorenzian dilaton profile $\nabla_{a} \Phi=0$. The region between two horizons corresponds to the interior of the black hole. As we increase $\gamma$, the interior region gets larger and larger. In general, the temperatures of the two horizons are different. However, as we saw in (5.7), in the large $\gamma$ limit, two temperatures eventually agree. Note again this solution is slightly different from the one in [40] by the choice of the sourceless part of the dilaton. If we choose the sourceless part as in [40], then the temperature of the two black holes always agree. However, this does not yield the reparametrization mode which satisfies the boundary conditions (3.7).

\section{Acknowledgments}

The author thanks Vijay Balasubramanian, Gábor Sárosi for useful discussions. TU was supported by JSPS Grant-in-Aid for Young Scientists 19K14716.

Open Access. This article is distributed under the terms of the Creative Commons Attribution License (CC-BY 4.0), which permits any use, distribution and reproduction in any medium, provided the original author(s) and source are credited.

\section{References}

[1] H. Umegaki, Conditional expectation in an operator algebra, iv (entropy and information), in Kodai Math. Sem. Rep., vol. 14, Department of Mathematics, Tokyo Institute of Technology, Tokyo Japan (1962), pp. 59-85 [DOI].

[2] A.C. Wall, A proof of the generalized second law for rapidly changing fields and arbitrary horizon slices, Phys. Rev. D 85 (2012) 104049 [Erratum ibid. 87 (2013) 069904] [arXiv:1105.3445] [INSPIRE].

[3] R. Bousso, H. Casini, Z. Fisher and J. Maldacena, Proof of a Quantum Bousso Bound, Phys. Rev. D 90 (2014) 044002 [arXiv: 1404.5635] [InSPIRE]. 
[4] T. Faulkner, R.G. Leigh, O. Parrikar and H. Wang, Modular Hamiltonians for Deformed Half-Spaces and the Averaged Null Energy Condition, JHEP 09 (2016) 038 [arXiv: 1605.08072] [INSPIRE].

[5] E. Witten, APS Medal for Exceptional Achievement in Research: Invited article on entanglement properties of quantum field theory, Rev. Mod. Phys. 90 (2018) 045003 [arXiv: 1803.04993] [INSPIRE].

[6] T. Faulkner, Bulk Emergence and the RG Flow of Entanglement Entropy, JHEP 05 (2015) 033 [arXiv: 1412.5648] [INSPIRE].

[7] S. Hollands and R.M. Wald, Stability of Black Holes and Black Branes, Commun. Math. Phys. 321 (2013) 629 [arXiv:1201.0463] [INSPIRE].

[8] N. Lashkari and M. Van Raamsdonk, Canonical Energy is Quantum Fisher Information, JHEP 04 (2016) 153 [arXiv: 1508.00897] [InSPIRE].

[9] S. Ryu and T. Takayanagi, Aspects of Holographic Entanglement Entropy, JHEP 08 (2006) 045 [hep-th/0605073] [INSPIRE].

[10] S. Ryu and T. Takayanagi, Holographic derivation of entanglement entropy from AdS/CFT, Phys. Rev. Lett. 96 (2006) 181602 [hep-th/0603001] [INSPIRE].

[11] V.E. Hubeny, M. Rangamani and T. Takayanagi, A Covariant holographic entanglement entropy proposal, JHEP 07 (2007) 062 [arXiv:0705.0016] [INSPIRE].

[12] G. Sárosi and T. Ugajin, Relative entropy of excited states in conformal field theories of arbitrary dimensions, JHEP 02 (2017) 060 [arXiv:1611.02959] [INSPIRE].

[13] G. Sárosi and T. Ugajin, Relative entropy of excited states in two dimensional conformal field theories, JHEP 07 (2016) 114 [arXiv: 1603.03057] [INSPIRE].

[14] G. Sárosi and T. Ugajin, Modular Hamiltonians of excited states, OPE blocks and emergent bulk fields, JHEP 01 (2018) 012 [arXiv:1705. 01486] [INSPIRE].

[15] T. Faulkner, F.M. Haehl, E. Hijano, O. Parrikar, C. Rabideau and M. Van Raamsdonk, Nonlinear Gravity from Entanglement in Conformal Field Theories, JHEP 08 (2017) 057 [arXiv: 1705.03026] [INSPIRE].

[16] N. Lashkari, H. Liu and S. Rajagopal, Modular Flow of Excited States, arXiv:1811.05052 [INSPIRE].

[17] J. Bhattacharya, M. Nozaki, T. Takayanagi and T. Ugajin, Thermodynamical Property of Entanglement Entropy for Excited States, Phys. Rev. Lett. 110 (2013) 091602 [arXiv: 1212.1164] [INSPIRE].

[18] D.D. Blanco, H. Casini, L.-Y. Hung and R.C. Myers, Relative Entropy and Holography, JHEP 08 (2013) 060 [arXiv: 1305.3182] [inSPIRE].

[19] G. Wong, I. Klich, L.A. Pando Zayas and D. Vaman, Entanglement Temperature and Entanglement Entropy of Excited States, JHEP 12 (2013) 020 [arXiv:1305.3291] [INSPIRE].

[20] F.M. Haehl, E. Hijano, O. Parrikar and C. Rabideau, Higher Curvature Gravity from Entanglement in Conformal Field Theories, Phys. Rev. Lett. 120 (2018) 201602 [arXiv: 1712.06620] [INSPIRE].

[21] T. Faulkner, M. Guica, T. Hartman, R.C. Myers and M. Van Raamsdonk, Gravitation from Entanglement in Holographic CFTs, JHEP 03 (2014) 051 [arXiv: 1312 .7856] [INSPIRE].

[22] D. Petz, Quasi-entropies for finite quantum systems, Rept. Math. Phys. 23 (1986) 57. 
[23] T. van Erven and P. Harremoës, Rényi divergence and kullback-leibler divergence, CoRR abs/1206.2459 (2012) [arXiv: 1206.2459].

[24] F. Brandão, M. Horodecki, N. Ng, J. Oppenheim and S. Wehner, The second laws of quantum thermodynamics, Proc. Nat. Acad. Sci. 112 (2015) 3275 [arXiv:1305.5278].

[25] H. Casini, R. Medina, I. Salazar Landea and G. Torroba, Renyi relative entropies and renormalization group flows, JHEP 09 (2018) 166 [arXiv: 1807.03305] [INSPIRE].

[26] M. Moosa, P. Rath and V.P. Su, A Rényi quantum null energy condition: proof for free field theories, JHEP 01 (2021) 064 [arXiv:2007.15025] [INSPIRE].

[27] J. de Boer, V. Godet, J. Kastikainen and E. Keski-Vakkuri, Quantum hypothesis testing in many-body systems, arXiv:2007.11711 [INSPIRE].

[28] N. Lashkari, Constraining Quantum Fields using Modular Theory, JHEP 01 (2019) 059 [arXiv: 1810.09306] [INSPIRE].

[29] T. Ugajin, Perturbative expansions of Rényi relative divergences and holography, JHEP 06 (2020) 053 [arXiv : 1812.01135] [INSPIRE].

[30] N. Bao, M. Moosa and I. Shehzad, The holographic dual of Rényi relative entropy, JHEP 08 (2019) 099 [arXiv: 1904.08433] [INSPIRE].

[31] A. May and E. Hijano, The holographic entropy zoo, JHEP 10 (2018) 036 [arXiv:1806.06077] [INSPIRE].

[32] A. Bernamonti, F. Galli, R.C. Myers and J. Oppenheim, Holographic second laws of black hole thermodynamics, JHEP 07 (2018) 111 [arXiv: 1803.03633] [INSPIRE].

[33] S. Balakrishnan and O. Parrikar, Modular Hamiltonians for Euclidean Path Integral States, arXiv: 2002.00018 [INSPIRE].

[34] R. Jackiw, Lower Dimensional Gravity, Nucl. Phys. B 252 (1985) 343 [inSPIRE].

[35] C. Teitelboim, Gravitation and hamiltonian structure in two spacetime dimensions, Phys. Lett. B 126 (1983) 41 [INSPIRE].

[36] A. Almheiri and J. Polchinski, Models of AdS 2 backreaction and holography, JHEP 11 (2015) 014 [arXiv: 1402.6334] [INSPIRE].

[37] J. Maldacena, D. Stanford and Z. Yang, Conformal symmetry and its breaking in two dimensional Nearly Anti-de-Sitter space, PTEP 2016 (2016) 12C104 [arXiv:1606.01857] [INSPIRE].

[38] G. Sárosi, $A d S_{2}$ holography and the SYK model, PoS Modave2017 (2018) 001 [arXiv: 1711.08482] [INSPIRE].

[39] D. Bak, C. Kim and S.-H. Yi, Bulk view of teleportation and traversable wormholes, JHEP 08 (2018) 140 [arXiv: 1805.12349] [INSPIRE].

[40] D. Bak, M. Gutperle and S. Hirano, Three dimensional Janus and time-dependent black holes, JHEP 02 (2007) 068 [hep-th/0701108] [INSPIRE].

[41] D. Bak, M. Gutperle and A. Karch, Time dependent black holes and thermal equilibration, JHEP 12 (2007) 034 [arXiv:0708.3691] [INSPIRE].

[42] J. Maldacena and D. Stanford, Remarks on the Sachdev-Ye-Kitaev model, Phys. Rev. D 94 (2016) 106002 [arXiv:1604.07818] [INSPIRE]. 
[43] V. Balasubramanian, A. Kar and T. Ugajin, Entanglement between two disjoint universes, JHEP 02 (2021) 136 [arXiv:2008.05274] [INSPIRE].

[44] Y. Chen, V. Gorbenko and J. Maldacena, Bra-ket wormholes in gravitationally prepared states, JHEP 02 (2021) 009 [arXiv: 2007.16091] [INSPIRE].

[45] A.M. García-García and V. Godet, Euclidean wormhole in the Sachdev-Ye-Kitaev model, Phys. Rev. D 103 (2021) 046014 [arXiv: 2010.11633] [InSPIRE]. 